\title{
Cytomegalovirus Infection and the Implications of Drug-Resistant Mutations in Pediatric Allogeneic Hematopoietic Stem Cell Transplant Recipients: A Retrospective Study from a Tertiary Hospital in China
}

Uet Yu (1) - Xiaodong Wang $\cdot$ Xiaoling Zhang $\cdot$ Chunjing Wang $\cdot$ Chunlan Yang

Xiaohui Zhou $\cdot$ Yue Li $\cdot$ Xiaochan Huang $\cdot$ Jing Wen $\cdot$ Feiqiu Wen $\cdot$ Sixi Liu

Accepted: April 28, 2021 / Published online: May 8, 2021

(c) The Author(s) 2021

\section{ABSTRACT}

Introduction: Drug-resistant cytomegalovirus (CMV) infection remains a challenge in the management of pediatric recipients of hematopoietic stem cell transplantation (HSCT). In this study, we retrospectively reviewed the clinical data on pediatric recipients of HSCT and identified known and unknown drug-resistant CMV variants.

Methods: A total of 221 children underwent allogeneic HSCT between October 2017 and November 2019 at Shenzhen Children's Hospital; of these, 35 patients were suspected of having drug-resistant CMV infections and were tested for drug-resistant mutations in the UL97 and UL54 genes by Sanger sequencing.

Supplementary Information The online version contains supplementary material available at https:// doi.org/10.1007/s40121-021-00452-4.

U. Yu $\cdot$ X. Wang $\cdot$ X. Zhang $\cdot$ C. Wang $\cdot$ C. Yang ·

X. Zhou · Y. Li · X. Huang · J. Wen - F. Wen .

S. Liu $(\square)$

Department of Hematology and Oncology,

Shenzhen Children's Hospital, Yitian Road No.

7019, Futian, Shenzhen 518038, China

e-mail: tiger647@126.com
Results: Mutations in UL97 or UL54, or in both, were detected in 11 patients. Most of these mutations have not been previously reported. The UL97 mutation (A582V) was detected in only one patient who also harbored two UL54 mutations (T760X and R876W). One patient with both the G604S and T691A mutations in the UL54 gene died of CMV pneumonia. We investigated the risk factors associated with the development of drug-resistant CMV infection. Patients in whom both the donor and recipient had positive CMV serostatuses were less likely to have drug-resistant mutations (Fisher's exact test, $p<0.05$ ).

Conclusion: Newly and previously detected CMV mutations in UL97 and UL54 may be associated with the development of drug-resistant CMV infection. The detection of these mutations may provide guidance for the management of post-transplant CMV infections.

Keywords: Cytomegalovirus; Hematopoietic stem cell transplantation; Pediatric; UL54; UL97 


\section{Key Summary Points}

Cytomegalovirus (CMV) infection is one of the most devastating complications and leading causes of pediatric recipients of allogeneic hematopoietic stem cell transplantation (HSCT).

Prolonged antiviral therapy may lead to the development of drug-resistance CMV variants.

This is one of the largest cohort studies investigating drug-resistant CMV mutations in pediatric HSCT recipients.

Some mutations identified in this study have been shown to have associations with antiviral drug susceptibilities or characterized as polymorphisms in previous literature. However, the roles of most of these mutations remain largely unknown and require further investigation.

The detection of drug-resistant CMV mutations may help to provide certain guidance for the management of posttransplant $\mathrm{CMV}$ infection in pediatric HSCT recipients.

\section{DIGITAL FEATURES}

This article is published with digital features, including a summary slide, to facilitate understanding of the article. To view digital features for this article go to https://doi.org/10.6084/ m9.figshare.14495763.

\section{INTRODUCTION}

Cytomegalovirus (CMV) infection remains one of the most devastating viral infections in recipients of hematopoietic stem cell transplantation (HSCT) [1]. Compared to adult patients, pediatric patients reportedly have higher risks of developing post-transplant CMV infections [2, 3]. Although end-organ diseases caused by CMV infection after HSCT are rare, $\mathrm{CMV}$ infections are associated with higher mortality, increased risks of developing complications at affected sites, and life-threatening infections with other pathogens [4, 5].

Currently, there are no standard guidelines for the prevention of CMV infection in pediatric recipients of HSCT. Antiviral drugs might be used as prophylactic, preemptive, or therapeutic strategies [2, 6]. However, the prolonged and repeated administration of antiviral drugs could cause resistance to these drugs, thereby resulting in treatment failure [7]. Therefore, the early identification of drug-resistant CMV variants is necessary to allow for appropriate therapeutic modifications in patients who do not have good response to standard treatment [8]. In this study, we retrospectively reviewed the clinical data on pediatric recipients of HSCT, and identified known and unknown drug-resistant CMV variants, focusing on mutations in the UL97 and UL54 genes, as well as their clinical relevance.

\section{METHODS}

\section{Patients}

A total of 221 patients who were younger than 18 years of age underwent allogeneic HSCT between October 2017 and November 2019 at the Shenzhen Children's Hospital, China. The follow-up period for at least 6 months following transplantation. Patient data, including clinical characteristics, treatments and outcomes, and laboratory test results, including donors' and recipients' CMV serostatuses, post-transplant plasma titers of CMV DNA, evidence of infections with other pathogens, and CMV genetic profiles were retrospectively reviewed. Written informed consents were obtained from parents or guardians for the collection, analyses, and publication of the patients' data. This study was approved by the Institutional Review Board of Shenzhen Children's Hospital (ethics approval number 202000302) and was conducted in 
accordance with the Helsinki Declaration of 1964 and its later amendments.

\section{Infection Monitoring}

The CMV serostatuses of donors and recipients were determined before HSCT. Plasma CMV DNA was monitored using real-time polymerase chain reaction (PCR) assay on a weekly basis for the first 3 months after HSCT, and at every outpatient visit thereafter. The CMV viral load was calibrated per the World Health Organization Standard for Human Cytomegalovirus for nucleic acid amplification techniques, NIBSC code 09/162 (1 IU/mL = 8 copies $/ \mathrm{mL})$ [9]. CMV DNAemia was defined as plasma levels of CMV DNA over $4 \times 10^{2}$ copies $/ \mathrm{mL} \quad(50 \mathrm{IU} / \mathrm{mL})$. Refractory CMV DNAemia was defined as persistent CMV DNA that was elevated by more than $1 \log 10$ or persistent viral load after 2 weeks, or less than $1 \log 10$ decline, or less than $1 \log 10$ elevation after 2 weeks of appropriately dosed antiviral therapy [10]. Recurrent CMV DNAemia was defined as CMV DNAemia at least 4 weeks after clearance of the previous infection. CMV diseases were diagnosed according to the clinical manifestations and the detection of CMV DNA at affected sites. Refractory CMV disease was defined as nonresolution of or lack of improvement in CMVrelated symptoms after 2 weeks of appropriate antiviral therapy [7, 11]. Patients with CMV diseases, those with refractory CMV DNAemia, or those suspected of having drug-resistant CMV infections based on clinical evaluations were tested for drug-resistant mutations (DRMs) in UL97 and UL54.

\section{Detection of DRMs in UL97 and UL54}

The DRMs in UL97 and UL54 were detected using Sanger sequencing [12]. CMV DNA for sequencing analysis was extracted from whole blood using an Aidlab total nucleic acid extraction kit (Aidlab Biotechnologies, Beijing, PRC) according to the manufacturer's instructions. Purified nucleic acids were then amplified to create amplicons spanning codons 420-640 of UL97 and codons 282-999 of UL54 using an
Applied Biosystems 7500 PCR amplifier (Applied Biosystems, Foster City, CA, USA). DNA was sequenced using an Applied Biosystems 3730XL DNA Analyzer (Applied Biosystems). Raw sequence data were analyzed using QIAGEN Variant Reporter software (version 1.1, QIAGEN, Redwood City, CA, USA).

\section{Treatment for CMV Infection}

Preemptive therapy was initiated in patients with plasma CMV DNA of at least $1 \times 10^{3}$ copies $/ \mathrm{mL}(125 \mathrm{IU} / \mathrm{mL})$ and in those with symptoms of CMV diseases, regardless of their CMV DNA level. Ganciclovir (GCV) was administered intravenously at $5 \mathrm{mg} / \mathrm{kg}$ every $12 \mathrm{~h}$ as first-line treatment. For patients suspected of having developed drug-resistant CMV infections, foscarnet (FOS) was administered intravenously at $60 \mathrm{mg} / \mathrm{kg}$ every $8 \mathrm{~h}$ with or without GCV. The course of antiviral treatment was guided by the monitoring of plasma CMV DNA titers, and the treatment continued until a negative plasma CMV DNA test result was obtained.

\section{Statistics}

Characteristics of patients with suspected drugresistant CMV infections were analyzed and compared between those with and without DRMs. Categorical variables were compared using Fisher's exact test. Continuous variables were compared using the Mann-Whitney $t$ test. A $p$ value less than 0.05 was considered statistically significant. All statistical analyses were performed using GraphPad Prism version 8 (GraphPad Software, San Diego, CA, USA).

\section{RESULTS}

\section{Patient Cohort and Detection of UL97 and UL54 Mutations}

A total of 221 pediatric patients received allogeneic HSCT between October 2017 and November 2019; of these, 112 had matched related or unrelated donors and 109 had 


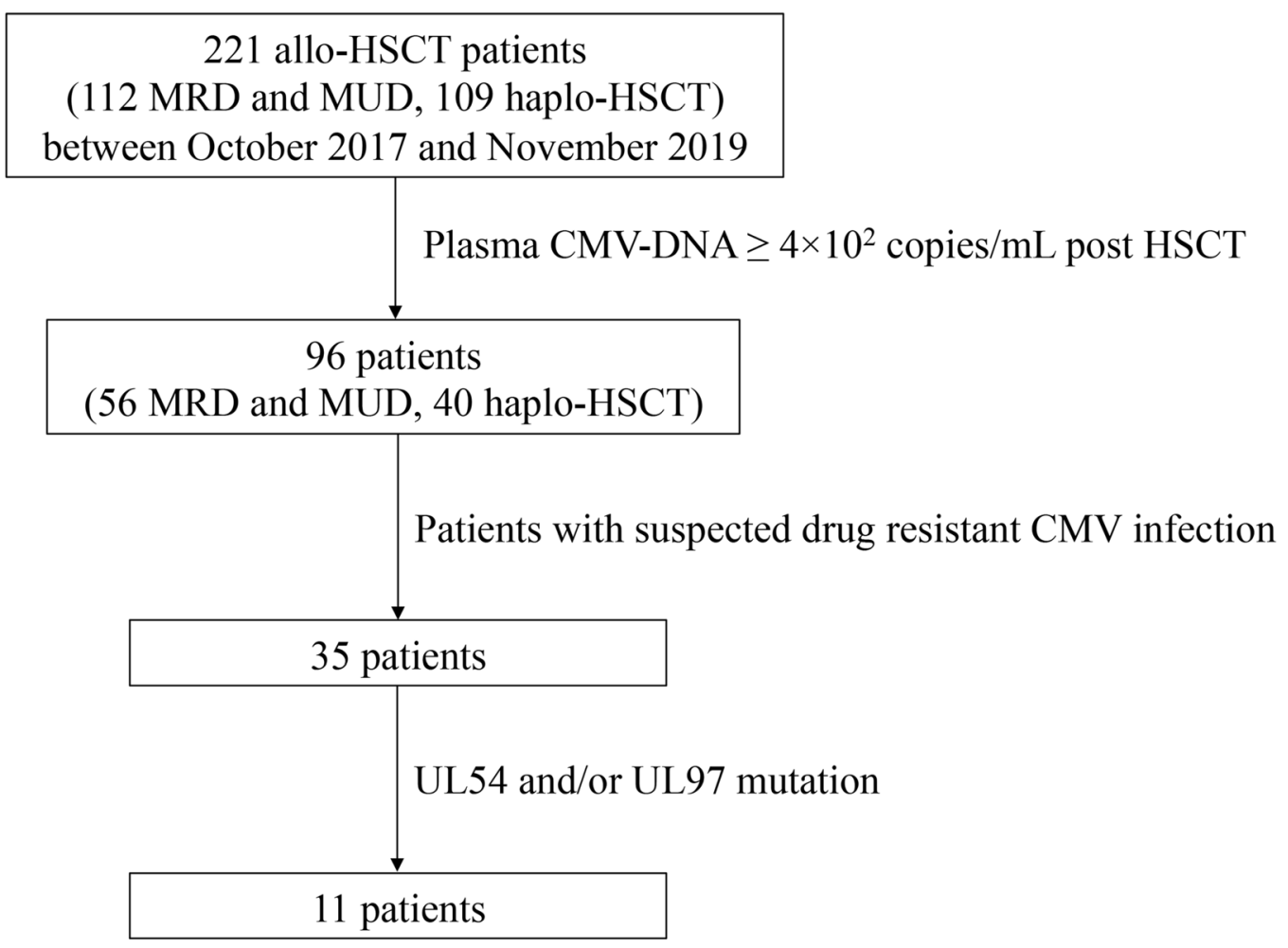

Fig. 1 Selection criteria of pediatric recipients of hematopoietic stem cell transplants that have been tested for mutations in the UL97 and UL54 genes by Sanger sequencing. Allo-HSCT allogeneic hematopoietic stem cell

haploidentical donors. CMV DNAemia (plasma CMV DNA greater than $4 \times 10^{2}$ copies $/ \mathrm{mL}$, or $50 \mathrm{IU} / \mathrm{mL}$ ) was observed in $96(43 \%)$ patients after HSCT. Thirty-five (16\%) patients were suspected of having developed drug-resistant CMV infections and were tested for DRMs in UL97 and UL54.

In order to identify the drug-resistant CMV variants, CMV DNA was extracted from all 35 patients with suspected drug-resistant infections and tested for mutations in UL97 and UL54 via Sanger sequencing (Fig. 1). As shown in Table 1, 11 of 35 patients harbored mutations in UL97 and UL54, either singly or in combination. Only one patient (patient 26) harbored a mutation (A582V) in UL97. This patient also harbored the T760X and R876W mutations in UL54. The other 10 patients harbored one or more mutations in UL54. The T691A mutation in UL54 was detected in patients 2 and 29. Patient 29 also harbored the UL54 G604S transplantation, MRD matched related donor, MUD matched unrelated donor, haplo-HSCT haploidentical hematopoietic stem cell transplantation

mutation and died as a result of CMV-related pneumonia. The A582V mutation in UL97 and the G604S, G633F, G678S, and T691A mutations in UL54 have been previously reported [12-18]. We additionally identified some novel UL54 mutations, namely E353Q, D514N, A614S, G677D, M727I, T760X, T885M, R876W, G878E, V973A, and L999I.

\section{Patient Characteristics}

Clinical data of patients with suspected drugresistant CMV infection is listed in Table 2. The age range was 1 to 14 years (median $7 \pm 3$ years), and most patients (71\%) were female. Sixty-nine percent of patients had haploidentical donors, and 31\% had matched related or unrelated donors. The CMV serostatuses were positive for both donors and recipients in most patients $(66 \%)$, and this was associated 
Table 1 Detection of mutations in UL97 and UL54 genes

\begin{tabular}{|c|c|c|c|c|c|c|}
\hline \multicolumn{4}{|l|}{ UL54 } & \multicolumn{3}{|l|}{ UL97 } \\
\hline $\begin{array}{l}\text { Patient } \\
\text { no. }\end{array}$ & Mutation & $\begin{array}{l}\text { Reported in } \\
\text { previous } \\
\text { literature }\end{array}$ & Drug resistance & Mutation & $\begin{array}{l}\text { Reported in } \\
\text { previous } \\
\text { literature }\end{array}$ & $\begin{array}{l}\text { Drug } \\
\text { resistance }\end{array}$ \\
\hline 2 & T691A & Yes $[17,18]$ & Polymorphism & & & \\
\hline 4 & A614S & No & Uncertain & & & \\
\hline 5 & G878E & No & Uncertain & & & \\
\hline \multirow[t]{3}{*}{13} & S633F & Yes $[17,18]$ & Polymorphism & & & \\
\hline & & & Uncertain & & & \\
\hline & V973A & No & & & & \\
\hline $19^{a}$ & L999I & No & Uncertain & & & \\
\hline \multirow[t]{2}{*}{21} & E353Q & No & Uncertain & & & \\
\hline & $\mathrm{D} 514 \mathrm{~N}$ & No & Uncertain & & & \\
\hline \multirow[t]{2}{*}{26} & T760X & No & Uncertain & $\mathrm{A} 582 \mathrm{~V}$ & Yes $[13,14]$ & Polymorphism \\
\hline & R876W & No & Uncertain & & & \\
\hline \multirow[t]{2}{*}{$29^{\mathrm{b}}$} & $\mathrm{T} 691 \mathrm{~A}$ & Yes $[17,18]$ & Polymorphism & & & \\
\hline & G604S & Yes [12] & Uncertain & & & \\
\hline 32 & M727I & No & Uncertain & & & \\
\hline \multirow[t]{2}{*}{34} & G678S & Yes $[15,16]$ & $\begin{array}{l}\text { Resistance to GCV and } \\
\text { CDV, sensitive to FOS }\end{array}$ & & & \\
\hline & $\mathrm{T} 885 \mathrm{M}$ & No & Uncertain & & & \\
\hline 35 & G677D & No & Uncertain & & & \\
\hline
\end{tabular}

$G C V$ ganciclovir, $C D V$ cidofovir, FOS foscarnet

a This patient developed one relapse of CMV DNAemia

b Deceased patient

with lower risks of developing DRMs after transplantation (Fisher's exact test, $\left.{ }^{*} p=0.0223\right)$. None of the patients had exposure to anti-CMV drugs before HSCT.

The primary diseases observed in the patients were thalassemia major (TM, 91\%), severe aplastic anemia (SAA, 6\%), and dyskeratosis congenita (DC, 3\%). Patients with TM received myeloablative conditioning chemotherapy, and those with SAA and DC received reduced-intensity conditioning chemotherapy. Prophylactic treatment for graft versus host disease (GVHD) included anti-thymocyte globulin, cyclosporine, tacrolimus, or mycophenolate mofetil. Four patients received rituximab because of a high anti-donor-specific antigen before HSCT. One patient received methylprednisolone to treat autoimmune hemolytic anemia, and another patient with TM received splenectomy before transplantation.

Median time to myeloid engraftment was $20 \pm 7$ days (mean \pm SD). Nine $(26 \%)$ patients developed acute GVHD (aGVHD), and six (17\%) patients developed grade II-IV aGVHD. Chronic GVHD (cGVHD) was observed in 4 (11\%) patients. However, only one of them developed 
Table 2 Characteristics of patients with suspected drug-resistant CMV infections

\begin{tabular}{|c|c|c|c|c|}
\hline \multirow[t]{2}{*}{ Patient characteristics } & \multicolumn{3}{|l|}{$n(\%)$, or mean $\pm \mathrm{SD}$} & \multirow[t]{2}{*}{$p$ value $^{\mathrm{a}}$} \\
\hline & Total patients & DRMs & No DRMs & \\
\hline Number of patients & 35 & $11(31 \%)$ & $24(69 \%)$ & - \\
\hline Female & $20 / 35(71 \%)$ & $8 / 11(73 \%)$ & $12 / 24(50 \%)$ & 0.2814 \\
\hline Age at transplant & $7 \pm 3$, range $1-14$ years & $7 \pm 2$ years & $8 \pm 3$ years & 0.6782 \\
\hline \multicolumn{5}{|l|}{ Primary disease } \\
\hline Thalassemia major & $32 / 35(91 \%)$ & $11 / 11(100 \%)$ & $21 / 24(88 \%)$ & 0.5361 \\
\hline Severe aplastic anemia & $2 / 35(6 \%)$ & $0 / 11$ & $2 / 24(8 \%)$ & - \\
\hline Dyskeratosis cogenita & $1 / 35(3 \%)$ & $0 / 11$ & $1 / 24(4 \%)$ & - \\
\hline Splenectomy ${ }^{\mathrm{b}}$ & $1 / 35(3 \%)$ & $0 / 11$ & $1 / 24(4 \%)$ & $>0.9999$ \\
\hline Graft type & & & & 0.4354 \\
\hline Matched donor & $11 / 35(31 \%)$ & $2 / 11(18 \%)$ & $9 / 24(38 \%)$ & - \\
\hline Haploidentical donor & $24 / 35(69 \%)$ & $9 / 11(82 \%)$ & $15 / 24(62 \%)$ & - \\
\hline \multicolumn{5}{|l|}{ Use of immunosuppressant before HSCT } \\
\hline rATG & $35 / 35(100 \%)$ & $11 / 11(100 \%)$ & $24 / 24(100 \%)$ & - \\
\hline Rituximab $^{c}$ & $4 / 35(11 \%)$ & $2 / 11(18 \%)$ & $2 / 24(8 \%)$ & 0.5748 \\
\hline Steroid $^{\mathrm{d}}$ & $1 / 35(3 \%)$ & $0 / 11$ & $1 / 24(4 \%)$ & $>0.9999$ \\
\hline aGVHD & $9 / 35(26 \%)$ & $4 / 11(36 \%)$ & $5 / 24(21 \%)$ & 0.4159 \\
\hline Grade II-IV aGVHD & $6 / 35(17 \%)$ & $2 / 11(18 \%)$ & $4 / 24(17 \%)$ & $>0.9999$ \\
\hline cGVHD & $4 / 35(11 \%)$ & $2 / 11(18 \%)$ & $2 / 24(8 \%)$ & 0.5748 \\
\hline Severe cGVHD & $1 / 35(3 \%)$ & $0 / 11$ & $1 / 24$ & $>0.9999$ \\
\hline Days to myeloid engraftment & $20 \pm 7$ days & $20 \pm 5$ days & $20 \pm 8$ days & 0.7537 \\
\hline Graft failure & $0 / 35$ & $0 / 11$ & $0 / 24$ & - \\
\hline \multicolumn{5}{|l|}{ CMV serostatus before HSCT } \\
\hline $\mathrm{D}-/ \mathrm{R}-$ & $4 / 35(11 \%)$ & $2 / 11(18 \%)$ & $2 / 24(8 \%)$ & 0.5748 \\
\hline $\mathrm{D}-/ \mathrm{R}+$ & $1 / 35(3 \%)$ & $1 / 11(9 \%)$ & $0 / 24$ & 0.3143 \\
\hline $\mathrm{D}+/ \mathrm{R}-$ & $7 / 35(20 \%)$ & $4 / 11(36 \%)$ & $3 / 24(13 \%)$ & 0.1715 \\
\hline $\mathrm{D}+/ \mathrm{R}+$ & $23 / 35(66 \%)$ & $4 / 11(36 \%)$ & $19 / 24(79 \%)$ & $0.0223^{*}$ \\
\hline Mismatched CMV serostatus & $8 / 35(23 \%)$ & $5 / 11(45 \%)$ & $3 / 24(13 \%)$ & 0.0767 \\
\hline Positive urine CMV DNA before HSCT & $10 / 35(29 \%)$ & $5 / 11(45 \%)$ & $5 / 24(21 \%)$ & 0.1344 \\
\hline Infection with other pathogens & $18 / 35(51 \%)$ & $5 / 11(45 \%)$ & $13 / 24(54 \%)$ & $>0.9999$ \\
\hline EBV DNAemia & $15 / 35(43 \%)$ & $4 / 11(36 \%)$ & $11 / 24(46 \%)$ & 0.4928 \\
\hline EBV-PTLD & $1 / 35(3 \%)$ & $0 / 11$ & $1 / 24(4 \%)$ & $>0.9999$ \\
\hline HSV-1 & $1 / 35(3 \%)$ & $0 / 11$ & $1 / 24(4 \%)$ & $>0.9999$ \\
\hline
\end{tabular}


Table 2 continued

\begin{tabular}{|c|c|c|c|c|}
\hline \multirow[t]{2}{*}{ Patient characteristics } & \multicolumn{3}{|c|}{$n(\%)$, or mean $\pm S D$} & \multirow[t]{2}{*}{$p$ value $^{\mathrm{a}}$} \\
\hline & Total patients & DRMs & No DRMs & \\
\hline $\mathrm{B} 19 \mathrm{~V}$ & $1 / 35(3 \%)$ & $1 / 11(9 \%)$ & $0 / 24$ & 0.3143 \\
\hline Tuberculosis & $1 / 35(3 \%)$ & $0 / 11$ & $1 / 24(4 \%)$ & $>0.9999$ \\
\hline
\end{tabular}

$D R M$ drug-resistant mutation, $H S C T$ hematopoietic stem cell transplantation, $r A T G$ rabbit anti-thymocyte globulin, a GVHD acute graft versus host disease, $c G V H D$ chronic graft versus host disease, $C M V$ cytomegalovirus, $E B V$ Epstein-Barr virus, PTLD post-transplant lymphoproliferative disease, $H S V-1$ type 1 herpes simplex virus, $B 19 V$ parvovirus B19 ${ }^{*} p<0.05$

${ }^{a}$ Fisher's exact test for categorical variables, Mann-Whitney $U$ test for continuous variables

b Splenectomy was performed in a patient with thalassemia major 2 years prior to HSCT

c Rituximab was given intravenously at $375 / \mathrm{m}^{2}$ per week for $2-4$ consecutive weeks to reduce the titer of donor-specific antibody before HSCT

d One patient received methylprednisolone intravenously and then orally for treatment of autoimmune hemolytic anemia before HSCT

severe cGVHD. Fifty-two percent of patients developed infection with other viruses. Epstein-Barr virus, type 1 herpes simplex virus, and parvovirus B19 infections were observed in $16(46 \%)$, one $(3 \%)$, and one $(3 \%)$ patients, respectively. One (3\%) patient developed pulmonary tuberculosis with CMV DNAemia (3\%). Co-infections with other bacteria or fungus were not observed. No other significant differences were observed between patients with or without DRMs.

\section{Infection and Treatment}

As shown in Table 3, the median interval from HSCT to the occurrence of CMV DNAemia was $42 \pm 13$ days (mean $\pm \mathrm{SD}$ ). The mean CMV DNA titer at the onset of DNAemia was $8.0 \pm 1.33 \times 10^{3}$ copies $/ \mathrm{mL}$ (mean $\pm \mathrm{SD}$ ) and increased by $10.83 \pm 30.01$-fold (mean \pm SD) at the peak titer. Interestingly, patients without DRMs had higher-fold, but not significant, increases in viral titers compared to those with DRMs. Additionally, patients without DRMs had a higher risks of developing CMV-related diseases. However, this difference was also not significant. Five (14\%) patients developed CMV diseases; four (11\%) developed CMV-related pneumonia and one (3\%) developed both CMVrelated pneumonia and retinitis.
Among the patients who received antiviral medications, 51\% received both GCV and FOS, and the remaining patients received either GCV $(29 \%)$ or FOS (17\%) alone. Notably, one patient received only intravenous immunoglobulin therapy without other antiviral medications. The patient tested negative for plasma CMV DNA at 18 days after the initial detection. Among the patients with refractory or persistent CMV diseases who did not show satisfactory responses to conventional treatment, three received transfusion of $\mathrm{CMV}$-specific cytotoxic T lymphocytes (CTLs). These patients showed reductions of CMV viral loads below 500 copies/ $\mathrm{mL}$ after two infusions of CTLs. No differences in treatment regimens were observed between patients with and without DRMs.

The durations of CMV DNAemia in patients with and without DRMs were $23 \pm 13$ and $29 \pm 18$ days, respectively. Five (14\%) patients experienced CMV DNAemia more than once. One patients with DRMs developed severe CMV-related pneumonia and died during the initial CMV infection. Another patient without DRMs had right eye blindness caused by CMV retinitis. The remaining patients recovered well after appropriate treatment with antiviral therapy. 
Table 3 Treatment and outcomes of patients with and without drug-resistant related mutations

\begin{tabular}{|c|c|c|c|c|}
\hline & \multicolumn{3}{|c|}{$n(\%)$, or mean \pm SD } & \multirow{2}{*}{$p$ value $^{\mathrm{a}}$} \\
\hline & Total patients & DRMs & No DRMs & \\
\hline Days to CMV DNAemia post $\mathrm{HSCT}^{\mathrm{b}}$ & $42 \pm 13$ days & $39 \pm 12$ days & $44 \pm 14$ days & 0.46 \\
\hline CMV DNA titer at onset (copies/mL) & $8.0 \pm 13.3 \times 10^{3}$ & $4.1 \pm 5.3 \times 10^{3}$ & $9.8 \pm 15.4 \times 10^{3}$ & 0.99 \\
\hline CMV DNA titer at onset $(\mathrm{IU} / \mathrm{mL})$ & $1.0 \pm 1.7 \times 10^{3}$ & $0.5 \pm 0.7 \times 10^{3}$ & $1.2 \pm 1.9 \times 10^{3}$ & 0.99 \\
\hline Peak CMV DNA titer increase by fold & $10.8 \pm 30.1$ & $2.0 \pm 2.0$ & $14.9 \pm 35.8$ & 0.24 \\
\hline CMV disease $^{c}$ & $5 / 35(14 \%)$ & $1 / 11(9 \%)$ & $4 / 24(17 \%)$ & 0.99 \\
\hline CMV pneumonia & $4 / 35(11 \%)$ & $1 / 11(9 \%)$ & $3 / 24(13 \%)$ & 0.99 \\
\hline $\mathrm{CMV}$ pneumonia + retinitis & $1 / 35(3 \%)$ & 0 & $1 / 24(4 \%)$ & - \\
\hline Duration to initiate antiviral treatment & 4 days ${ }^{\mathrm{d}}$ & 4 days & 3 days $^{\mathrm{d}}$ & 0.48 \\
\hline \multicolumn{5}{|l|}{ Treatment } \\
\hline GCV & $10 / 35(29 \%)$ & $2 / 11(18 \%)$ & $8 / 24(33 \%)$ & 0.43 \\
\hline FOS & $6 / 35(17 \%)$ & $2 / 11(18 \%)$ & $4 / 24(17 \%)$ & $>0.99$ \\
\hline GCV + FOS & $18 / 35(51 \%)$ & $7 / 11(64 \%)$ & $11 / 24(46 \%)$ & 0.47 \\
\hline No antiviral treatment & $1 / 35(3 \%)$ & 0 & $1 / 24(4 \%)$ & - \\
\hline CTL & $3 / 35(8 \%)$ & $1 / 11(9 \%)$ & $2 / 24(8 \%)$ & $>0.99$ \\
\hline Duration of CMV DNAemia $^{e}$ & $28 \pm 17$ days & $23 \pm 13$ days & $29 \pm 18$ days & 0.3845 \\
\hline \multicolumn{5}{|l|}{ Episodes of CMV DNAemia } \\
\hline 1 episode & $30 / 35(86 \%)$ & $10 / 11(91 \%)$ & $20 / 24(83 \%)$ & $>0.9999$ \\
\hline 2 episodes & $4 / 35(8 \%)$ & $1 / 11(9 \%)$ & $3 / 24(13 \%)$ & $>0.9999$ \\
\hline 3 episodes & $1 / 35(3 \%)$ & $0 / 11$ & $1 / 24(4 \%)$ & - \\
\hline Death $^{\mathrm{f}}$ & $1 / 35(3 \%)$ & $1 / 11(9 \%)$ & 0 & - \\
\hline Alive with complications ${ }^{\mathrm{g}}$ & $1 / 35(3 \%)$ & 0 & $1 / 24(4 \%)$ & - \\
\hline
\end{tabular}

DRM drug-resistant related mutation, $C M V$ cytomegalovirus, HSCT hematopoietic stem cell transplantation, $G C V$ ganciclovir, FOS foscarnet, $C T L$ cytotoxic T lymphocyte

${ }^{a}$ Fisher's exact test for categorical variables, Mann-Whitney $t$ test for continuous variables

${ }^{b}$ Four patients developed CMV DNAemia during prophylactic treatment with foscarnet

c All patients with confirmed CMV diseases had CMV pneumonia

${ }^{d}$ Excluding one patient that did not receive treatment with antiviral drugs

e Plasma CMV DNA was assessed by real-time polymerase chain reaction assay

${ }^{\mathrm{f}}$ Patient died of severe respiratory failure caused by CMV pneumonia

g This patient had blindness of the right eye caused by CMV retinitis and chronic GVHD

\section{DISCUSSION}

In our study, or the 221 recipients of allogeneic HSCT, we identified 35 patients with suspected drug-resistant CMV infection. As prolonged and repeated antiviral treatment for CMV infections could result in the development of drug-resistant viral variants, patients with suspected drug- 
resistant CMV infections should be screened for DRMs to allow for early interventions [7, 11]. In the current study, patients did not have antiCMV treatment before HSCT. However, patients with positive CMV statuses suggested prior exposure to CMV before transplant. Post-transplant CMV status was monitored by screening plasma CMV DNA levels on a weekly basis for the first 3 months after HSCT, and then at every return visit thereafter. Patients were considered to have CMV DNAemia if their plasma CMV DNA levels were at least 400 copies/mL (50 IU/ $\mathrm{mL}$ ). However, preemptive antiviral therapies were administered only if the patients had rising plasma CMV DNA levels of at least 1000 copies/mL $(125 \mathrm{IU} / \mathrm{mL})$ or had developed symptoms suggestive of CMV diseases, regardless of viral load.

We examined DRMs by identifying mutations in UL97 and UL54 in patients with drugresistant CMV infections. GCV-resistant mutations are observed more frequently in UL97 $[19,20]$. In clinical isolates, known CMV-resistant mutations are mainly located in the highly conserved region between codons 439 and 641 [21]. Less frequently, GCV resistance results from mutations in the DNA polymerase UL54. Mutations in UL54 also confer resistance to most known DNA polymerase inhibitors, including GCV, valaciclovir (VACV), FOS, and cidofovir (CDV) $[22,23]$. Other drug-resistant CMV variants, including those with mutations in the CMV DNA terminase complex members UL56, UL89, and UL51, are known to confer reduced susceptibility to antivirals such as letermovir (LTV) [19, 24-26]. However, mutations in UL89 and UL54 are only observed under in vitro selection pressure with drugs. Identification of these CMV variants in clinical isolates and their relevance to patient outcomes require further investigation.

GCV remains the first-line treatment for post-HSCT CMV infection. Some of the known mutations in UL97 confer a high level of GCV resistance, and in such cases, the use of alternative drugs is recommended [27, 28]. However, it is difficult to determine the optimal antiviral therapy in patients with mutations that confer low-level GCV resistance or new mutations with unknown effects on drug sensitivity [19].
Inadequate dosing or differences in drug concentrations at infected sites could contribute to treatment failure [29]. In this study, patients received GCV at a full dose of $5 \mathrm{mg} / \mathrm{kg}$ every $12 \mathrm{~h}$. Some studies recommended that recipients of solid organ transplants that show lowlevel GCV resistance may be treated with an escalation of GCV doses up to $10 \mathrm{mg} / \mathrm{kg}$ every $12 \mathrm{~h}$, but this could increase the risks of renal toxicity [30]. Thus, close monitoring of the pharmacokinetics of antivirals and renal functions in patients is required if this therapeutic strategy is applied to pediatric recipients of HSCT in the future.

In this study, more than half of the patients with suspected drug-resistant CMV infections were administered FOS with GCV. Additionally, FOS monotherapy was administered in six patients who had risk factors associated with the development of drug-resistant CMV infection, such as receiving of a haploidentical graft, incompatible CMV serostatuses between the donor and the recipient, GVHD, and the intensity of immunosuppressive therapy. Therapy with FOS was not associated with a higher risks of developing DRMs. CDV is generally considered as a third-line treatment for postHSCT CMV infections [7]. Recently, LTV has been well accepted as prophylaxis in patients with HSCT [31]. Other novel antiviral agents, including maribavir, brincidofovir, and leflunomide, are being evaluated for their safety and treatment efficacies for CMV infections in HSCT recipients [32-34]. However, none of these novel antiviral drugs is currently approved in China; hence, this limits the treatment options for patients in this study. In the current study, patients with refractory or resistant CMV diseases that did not respond to conventional treatment were transfused of CMV-specific CTLs and achieved good responses. Previous studies have demonstrated that CTLs may provide protective immunity for both early and late CMV infections, and these have been applied as first-line treatment combined with conventional antivirals against CMV infection in HSCT recipients in a recent clinical trial $[35,36]$.

Eleven (5\%) patients harbored mutations in UL97 and UL54. However, most of the DRMs detected in this study have not been previously 
identified in clinical samples; hence, their effects on drug sensitivity remain uncertain. Only one patient (patient 26) harbored the UL97 (A582V) mutation. This mutation has been previously reported in blood samples from solid organ transplant recipients. However, in vitro sensitivity analyses suggested that this CMV variant was sensitive to GCV $[13,14]$. Additionally, this patient was found to harbor the T760X and R876W mutations in UL54. Whether the combination of mutations in these genes can decrease drug sensitivity remains unknown, because this patient achieved a favorable outcome and did not develop CMVrelated diseases or relapse after antiviral treatment.

Ten patients harbored UL54 mutations, including the E353Q, D514N, G557D, G604S, A614S, G678S, M727I, G878E, T885M, V973A, and L999I mutations. Of these, only the G604S, S633F, G678S, and T691A mutations have been reported previously $[12,15-17]$. Our study is the first to report the other mutations, which should be added to the extensive list of known UL54 mutations that confer resistance to multiple antivirals. The T691A mutation was found in two patients (patients 2 and 29). Together with the S633F mutation, these mutations have been characterized as polymorphisms in previous literature [17]. Patient 29 also harbored the G604S mutation in UL54. These two patients had distinct outcomes. Patient 2 responded well to antivirals, whereas patient 29 died of severe CMV-related pneumonia 44 days after the initiation of antiviral therapy. Our data suggest that this mutation may confer resistance to anti-CMV treatment. However, the exact role of the G604S mutation remains uncertain because this has been previously observed in clinical isolates from recipients of solid organ transplantation and characterized with unknown significance [12]. Combinations of mutations in the UL54 gene are known to increase the level of drug resistance $[37,38]$. Additionally, in vitro studies have suggested that different mutations in genetic loci with low-grade resistance to LTV may combine to cause high-grade resistance or promote other mutations having the same effects [25]. Although we suspect that combined G604S and T691A mutations in UL54 may lead to a multiplier effect on the suppression of antiviral therapies, the exact correlation and impact on treatment outcomes require further investigation.

We compared the clinical characteristics and patient outcomes between patients with and without drug-resistant CMV mutations. Among patients with CMV diseases or those with more than one episode of CMV DNAemia, only two patients harbored drug-resistant CMV variants (patients 19 and 29) (Table 1 in the supplementary material). However, we could have underestimated the number of DRMs as a result of the sensitivity of Sanger sequencing. A mutation can only be detected with Sanger sequencing if it is present in at least $20-30 \%$ of the viral population [39]. Additionally, we only assessed mutations in codons 420-640 in UL97 and codons 282-999 in UL54. Although rarely reported, the existence of CMV mutations outside these regions is hitherto unknown. Future studies using deep sequencing may help to identify unknown mutations affecting the susceptibility to antiviral therapies [40, 41]. Another limitation is that we were not able to examine the pharmacokinetics of antivirals used in this study; hence, antiviral dosing for patients with low levels of DRM resistance may not have been optimized. Additionally, we were unable to find a significant correlation between the presence of DRMs and the development of drug-resistant CMV infections because of the low incidence of CMV infection.

\section{CONCLUSIONS}

Our data suggest that DRMs in CMV may have an impact on the drug susceptibility and outcomes of pediatric recipients of HSCT. The detection of these mutations may provide guidance for management of post-transplant CMV infections. However, further analysis is required to confirm the exact clinical correlation between these mutations and disease outcomes. 


\section{ACKNOWLEDGEMENTS}

We thank Shanghai Tissuebank Diagnostics for performing the Sanger sequencing. We thank Dr. Kuang-Yueh Chiang at the Hospital for Sick Children in Toronto, Canada for the mentorship and parents and patients that participated in this study.

Funding. This study was funded by Sanming Project of Medicine in Shenzhen (SZSM 201512033), Shenzhen Fund for Guangdong Provincial High-level Clinical Key Specialties (SZGSP012), Shenzhen Science and Technology Program (RCBS20200714114858018), Shenzhen Key Medical Discipline Construction Fund (SZXK034) for study design and data analysis. The journal's Rapid Service Fee is funded by Shenzhen Healthcare Research Project (SZLY2018015) and Shenzhen Children's Hospital Research Fund (ynkt2020-zz01).

Authorship. All named authors meet the International Committee of Medical Journal Editors (ICMJE) criteria for authorship for this article, take responsibility for the integrity of the work as a whole, and have given their approval for this version to be published.

Author Contributions. Uet Yu provided the conceptualization of this study and wrote this manuscript. Xiaodong Wang, Xiaoling Zhang, Chunjing Wang, Chunlan Yang, Xiaohui Zhou, Yue Li, Xiaochan Huang, and Jing Wen conducted the investigation and validation of data. Feiqiu Wen gave critical reviews to this manuscript. Sixi Liu supervised this study and gave critical reviews to this manuscript.

Prior Publication. Data of this paper has been presented in an oral presentation at the 52nd Congress of the International Paediatric Oncology (SIOP) Virtual Congress, 14-17 October 2020.

Disclosures. The authors (Uet Yu, Xiaodong Wang, Xiaoling Zhang, Chunjing Wang, Chunlan Yang, Xiaohui Zhou, Yue Li, Xiaochan Huang, Jing Wen, Feiqiu Wen, Sixi Liu) declare no conflict of interests
Compliance with Ethics Guidelines. This study was approved by the Institutional Review Board of Shenzhen Children's Hospital (ethics approval number 202000302) and was conducted in accordance with the Helsinki Declaration of 1964 and its later amendments. Written informed consents were obtained from parents or guardians for the collection, analyses, and publication of the patients' data.

Data Availability. The datasets generated during and/or analyzed during the current study are available from the corresponding author on reasonable request.

Open Access. This article is licensed under a Creative Commons Attribution-NonCommercial 4.0 International License, which permits any non-commercial use, sharing, adaptation, distribution and reproduction in any medium or format, as long as you give appropriate credit to the original author(s) and the source, provide a link to the Creative Commons licence, and indicate if changes were made. The images or other third party material in this article are included in the article's Creative Commons licence, unless indicated otherwise in a credit line to the material. If material is not included in the article's Creative Commons licence and your intended use is not permitted by statutory regulation or exceeds the permitted use, you will need to obtain permission directly from the copyright holder. To view a copy of this licence, visit http:// creativecommons.org/licenses/by-nc/4.0/.

\section{REFERENCES}

1. Zanella MC, Cordey S, Kaiser L. Beyond cytomegalovirus and Epstein-Barr virus: a review of viruses composing the blood virome of solid organ transplant and hematopoietic stem cell transplant recipients. Clin Microbiol Rev. 2020. https://doi. org/10.1128/cmr.00027-20.

2. Wattles BA, Kim AJ, Cheerva AC, Lucas KG, Elder JJ. Cytomegalovirus treatment in pediatric hematopoietic stem cell transplant patients. J Pediatr Hematol Oncol. 2017;39(4):241-8. https://doi. org/10.1097/mph.0000000000000730. 
3. Czyżewski K, Styczyński J, Giebel S, et al. Age-dependent determinants of infectious complications profile in children and adults after hematopoietic cell transplantation: lesson from the nationwide study. Ann Hematol. 2019;98(9):2197-211. https:// doi.org/10.1007/s00277-019-03755-2.

4. Pande A, Dubberke ER. Cytomegalovirus infections of the stem cell transplant recipient and hematologic malignancy patient. Infect Dis Clin $\mathrm{N}$ Am. 2019;33(2):485-500. https://doi.org/10.1016/j.idc. 2019.02.008.

5. Piret J, Schibler M, Pham VD, et al. Compartmentalization of a multidrug-resistant cytomegalovirus UL54 mutant in a stem cell transplant recipient with encephalitis. J Infect Dis. 2019;220(8):1302-6. https://doi.org/10.1093/infdis/jiz298.

6. Bontant T, Sedlaçek P, Balduzzi A, et al. Survey of CMV management in pediatric allogeneic HSCT programs, on behalf of the inborn errors, infectious diseases and pediatric diseases working parties of EBMT. Bone Marrow Transpl. 2014;49(2):276-9. https://doi.org/10.1038/bmt.2013.164.

7. Ljungman $\mathrm{P}$, de la Camara $\mathrm{R}$, Robin $\mathrm{C}$, et al. Guidelines for the management of cytomegalovirus infection in patients with haematological malignancies and after stem cell transplantation from the 2017 European Conference on Infections in Leukaemia (ECIL 7). Lancet Infect Dis. 2019;19(8): e260-72. https://doi.org/10.1016/s14733099(19)30107-0.

8. Danziger-Isakov L, Englund J, Green M, PosfayBarbe KM, Zerr DM. Cytomegalovirus in pediatric hematopoietic stem cell transplantation: a casebased panel discussion of current challenges. J Pediatr Infect Dis Soc. 2018;7(Suppl_2):72-4. https:// doi.org/10.1093/jpids/piy104.

9. Fryer JF, Heath AB, Minor PD. A collaborative study to establish the 1st WHO International Standard for human cytomegalovirus for nucleic acid amplification technology. Biologicals. 2016;44(4):242-51. https://doi.org/10.1016/j.biologicals.2016.04.005.

10. Chemaly RF, Chou S, Einsele H, et al. Definitions of resistant and refractory cytomegalovirus infection and disease in transplant recipients for use in clinical trials. Clin Infect Dis. 2019;68(8):1420-6. https://doi.org/10.1093/cid/ciy696.

11. Razonable RR, Humar A. Cytomegalovirus in solid organ transplant recipients-guidelines of the American Society of Transplantation Infectious Diseases Community of Practice. Clin Transpl. 2019;33(9):e13512. https://doi.org/10.1111/ctr. 13512 .
12. López-Aladid R, Guiu A, Sanclemente G, et al. Detection of cytomegalovirus drug resistance mutations in solid organ transplant recipients with suspected resistance. J Clin Virol. 2017;90:57-63. https://doi.org/10.1016/j.jcv.2017.03.014.

13. Boivin G, Goyette N, Gilbert C, et al. Absence of cytomegalovirus-resistance mutations after valganciclovir prophylaxis, in a prospective multicenter study of solid-organ transplant recipients. J Infect Dis. 2004;189(9):1615-8. https://doi.org/10.1086/ 382753.

14. Martin M, Gilbert C, Covington E, Boivin G. Characterization of human cytomegalovirus (HCMV) UL97 mutations found in a valganciclovir/ oral ganciclovir prophylactic trial by use of a bacterial artificial chromosome containing the HCMV genome. J Infect Dis. 2006;194(5):579-83. https:// doi.org/10.1086/505882.

15. Smith IL, Taskintuna I, Rahhal FM, et al. Clinical failure of CMV retinitis with intravitreal cidofovir is associated with antiviral resistance. Arch Ophthalmol. 1998;116(2):178-85. https://doi.org/10.1001/ archopht.116.2.178.

16. Erice A. Resistance of human cytomegalovirus to antiviral drugs. Clin Microbiol Rev. 1999;12(2): 286-97.

17. Lurain NS, Chou S. Antiviral drug resistance of human cytomegalovirus. Clin Microbiol Rev. 2010;23(4):689-712. https://doi.org/10.1128/cmr. 00009-10.

18. Fillet AM, Auray L, Alain S, et al. Natural polymorphism of cytomegalovirus DNA polymerase lies in two nonconserved regions located between domains delta-C and II and between domains III and I. Antimicrob Agents Chemother. 2004;48(5): 1865-8. https://doi.org/10.1128/aac.48.5.18651868.2004.

19. Razonable RR. Drug-resistant cytomegalovirus: clinical implications of specific mutations. Curr Opin Organ Transpl. 2018;23(4):388-94. https:// doi.org/10.1097/mot.0000000000000541.

20. Marfori JE, Exner MM, Marousek GI, Chou S, Drew WL. Development of new cytomegalovirus UL97 and DNA polymerase mutations conferring drug resistance after valganciclovir therapy in allogeneic stem cell recipients. J Clin Virol. 2007;38(2):120-5. https://doi.org/10.1016/j.jcv.2006.11.005.

21. Kim YJ, Boeckh M, Cook L, et al. Cytomegalovirus infection and ganciclovir resistance caused by UL97 mutations in pediatric transplant recipients. Transpl Infect Dis. 2012;14(6):611-7. https://doi. org/10.1111/j.1399-3062.2012.00760.x. 
22. Schnepf N, Dhédin N, Mercier-Delarue S, et al. Dynamics of cytomegalovirus populations harbouring mutations in genes UL54 and UL97 in a haematopoietic stem cell transplant recipient. J Clin Virol. 2013;58(4):733-6. https://doi.org/10. 1016/j.jcv.2013.10.007.

23. Choi SH, Hwang JY, Park KS, et al. The impact of drug-resistant cytomegalovirus in pediatric allogeneic hematopoietic cell transplant recipients: a prospective monitoring of UL97 and UL54 gene mutations. Transpl Infect Dis. 2014;16(6):919-29. https://doi.org/10.1111/tid.12311.

24. Douglas CM, Barnard R, Holder D, et al. Letermovir resistance analysis in a clinical trial of cytomegalovirus prophylaxis for hematopoietic stem cell transplant recipients. J Infect Dis. 2020;221(7): 1117-26. https://doi.org/10.1093/infdis/jiz577.

25. Chou S, Satterwhite LE, Ercolani RJ. New locus of drug resistance in the human cytomegalovirus UL56 gene revealed by in vitro exposure to letermovir and ganciclovir. Antimicrob Agents Chemother. 2018. https://doi.org/10.1128/aac.0092218.

26. Gerna G, Lilleri D, Baldanti F. An overview of letermovir: a cytomegalovirus prophylactic option. Expert Opin Pharmacother. 2019;20(12):1429-38. https://doi.org/10.1080/14656566.2019.1637418.

27. Hantz S, Garnier-Geoffroy F, Mazeron MC, et al. Drug-resistant cytomegalovirus in transplant recipients: a French cohort study. J Antimicrob Chemother. 2010;65(12):2628-40. https://doi.org/10. $1093 / j a c / d k q 368$.

28. Avery RK, Arav-Boger R, Marr KA, et al. Outcomes in transplant recipients treated with foscarnet for ganciclovir-resistant or refractory cytomegalovirus infection. Transplantation. 2016;100(10):e74-80. https://doi.org/10.1097/tp.0000000000001418.

29. Baillie GM. Pharmacokinetics of antiviral agents for the treatment of cytomegalovirus infection. Am J Health Syst Pharm. 2005;62(8 Suppl 1):S14-7. https://doi.org/10.1093/ajhp/62.suppl_1.S14.

30. Kotton CN, Kumar D, Caliendo AM, et al. The third international consensus guidelines on the management of cytomegalovirus in solid-organ transplantation. Transplantation. 2018;102(6):900-31. https://doi.org/10.1097/tp.0000000000002191.

31. Sassine J, Khawaja F, Shigle TL, et al. Refractory and resistant cytomegalovirus after hematopoietic cell transplant in the letermovir primary prophylaxis era. Clin Infect Dis. 2021. https://doi.org/10.1093/ cid/ciab298.
32. Imlay HN, Kaul DR. Letermovir and maribavir for the treatment and prevention of cytomegalovirus infection in solid organ and stem cell transplant recipients. Clin Infect Dis. 2020. https://doi.org/10. 1093/cid/ciaa1713.

33. Marty FM, Winston DJ, Chemaly RF, et al. A randomized, double-blind, placebo-controlled phase 3 trial of oral brincidofovir for cytomegalovirus prophylaxis in allogeneic hematopoietic cell transplantation. Biol Blood Marrow Transpl. 2019;25(2): 369-81. https://doi.org/10.1016/j.bbmt.2018.09. 038.

34. Gokarn A, Toshniwal A, Pathak A, et al. Use of leflunomide for treatment of cytomegalovirus infection in recipients of allogeneic stem cell transplant. Biol Blood Marrow Transpl. 2019;25(9): 1832-6. https://doi.org/10.1016/j.bbmt.2019.04. 028.

35. Zhao XY, Pei XY, Chang YJ, et al. First-line therapy with donor-derived human cytomegalovirus (HCMV)-specific T cells reduces persistent HCMV infection by promoting antiviral immunity after allogenic stem cell transplantation. Clin Infect Dis. 2020;70(7):1429-37. https://doi.org/10.1093/cid/ ciz368.

36. Luo XH, Huang XJ, Liu KY, Xu LP, Liu DH. Protective immunity transferred by infusion of cytomegalovirus-specific CD8(+) T cells within donor grafts: its associations with cytomegalovirus reactivation following unmanipulated allogeneic hematopoietic stem cell transplantation. Biol Blood Marrow Transpl. 2010;16(7):994-1004. https://doi. org/10.1016/j.bbmt.2010.02.007.

37. Chou S, Marousek GI, Van Wechel LC, Li S, Weinberg A. Growth and drug resistance phenotypes resulting from cytomegalovirus DNA polymerase region III mutations observed in clinical specimens. Antimicrob Agents Chemother. 2007;51(11): 4160-2. https://doi.org/10.1128/aac.00736-07.

38. Drouot E, Piret J, Lebel MH, Boivin G. Characterization of multiple cytomegalovirus drug resistance mutations detected in a hematopoietic stem cell transplant recipient by recombinant phenotyping. J Clin Microbiol. 2014;52(11):4043-6. https://doi. org/10.1128/jcm.02205-14.

39. Zürcher S, Mooser C, Lüthi AU, et al. Sensitive and rapid detection of ganciclovir resistance by PCR based MALDI-TOF analysis. J Clin Virol. 2012;54(4): 359-63. https://doi.org/10.1016/j.jcv.2012.04.019.

40. Cunha-Bang C, Kirkby N, Sønderholm M, et al. The time course of development and impact from viral resistance against ganciclovir in cytomegalovirus infection. Am J Transpl. 2013;13(2):458-66. https:// doi.org/10.1111/ajt.12042. 
41. Sahoo MK, Lefterova MI, Yamamoto F, et al. Detection of cytomegalovirus drug resistance mutations by next-generation sequencing. J Clin
Microbiol. 2013;51(11):3700-10. https://doi.org/ 10.1128/jcm.01605-13. 